\title{
Enhancement of Anti-Hypoxic Activity and Differentiation of Cardiac Stem Cells by Supernatant Fluids from Cultured Macrophages that Phagocytized Dead Mesenchymal Stem Cells
}

\author{
Liang Liu ${ }^{1,+}$, Xian Jin ${ }^{2,+}$, Zhong'e Zhou ${ }^{2}$ and Chengxing Shen ${ }^{1, *}$ \\ 1 Department of Cardiology, Xinhua Hospital, Shanghai Jiao Tong University School of Medicine, \\ 1665 Kongjiang Road, Shanghai 200092, China; liuliangsuda@163.com (L.L.) \\ 2 Department of Cardiology, Central Hospital of Minhang District, 170 Xinsong Road, Shanghai 201199, China; \\ jinxianian@126.com (X.J.); zhouzhonge2006@126.com (Z.Z.) \\ * Correspondence: shencx@sjtu.edu.cn; Tel./Fax: +86-21-2507-7300 \\ + These authors contributed equally to this work.
}

Academic Editor: Maurizio Battino

Received: 14 June 2016; Accepted: 13 July 2016; Published: 20 July 2016

\begin{abstract}
Background: Most mesenchymal stem cells (MSCs) die shortly after transplantation into a myocardial infarcted area. Dead MSCs (dMSCs) are phagocytized by macrophages (pM $\Phi)$ in vivo and in vitro; however, the effects of $\mathrm{pM} \Phi$ on cardiac stem cells (CSCs) remain unknown. Methods: MSCs, CSCs, and macrophages were obtained from bone marrow, hearts, and peritoneal cavity of mice, respectively. dMSCs were harvested after hypoxia for $24 \mathrm{~h}$, and incubated with macrophages (2:1) for another 2 days with or without lipopolysaccharide (LPS, $50 \mathrm{ng} / \mathrm{mL}$ ) and sorted by flow cytometry to obtain $\mathrm{pM} \Phi$. Viability and apoptosis of CSCs were respectively evaluated with the cell counting kit-8 (CCk-8) assay and Annexin V-PE/7-AAD staining at 0,6, 12, and $24 \mathrm{~h}$ of culture with supernatant fluids from macrophages (M $\Phi)$, LPS-stimulated macrophages (LPS-pM $\Phi), \mathrm{pM} \Phi$, and MSCs. GATA-4 and c-TnI expression was measured by flow cytometry on the seventh day. Expression of inflammation and growth factors was assessed by real-time polymerase chain reaction (RT-PCR) in M $\Phi$, LPS-pM $\Phi$, and $\mathrm{pM} \Phi$ cells. Results: $\mathrm{pM} \Phi$ expressed higher levels of interleukin-10 (IL-10) and transforming growth factor- $\beta$ (TGF- $\beta$ )and lower levels of tumor necrosis factor- $\alpha$ (TNF- $\alpha$ )and IL- 6 than LPS-pM $\Phi$, higher levels of growth factors and of GATA-4 and c-TnI at the 7th day, which were similar to those in MSCs. CSCs cultured with supernatant fluids of $\mathrm{pM} \Phi$ exhibited higher proliferative, anti-hypoxic, and differentiation activities. Conclusion: The supernatant fluids of macrophages that had phagocytized dead MSCs encouraged changes in phenotype and growth factor expression, enhanced proliferation, differentiation, and anti-hypoxic activity of CSCs, which is relevant to understanding the persistent therapeutic effect of MSCs after their massive demise upon transplantation in myocardial infarction. Furthermore, some miRNAs or proteins which were extracted from the supernatant fluids may give us a new insight into the treatment of myocardial infarction in the future.
\end{abstract}

Keywords: macrophages; dMSC; CSCs; growth factors; differentiation

\section{Introduction}

Cardiovascular disease, especially myocardial infarction, remains the leading cause of mortality and morbidity around the world [1] and the focus of intense clinical and basic research-in particular on transplantation of stem and progenitor cells to rescue the injured myocardium [2,3]. 
Bone marrow-derived mesenchymal stem cells (MSCs), a type of multipotent stem cell that can differentiate into various cell types, such as osteoblasts, muscle cells, and cardiomyocytes $[4,5]$, have unique immunological characteristics that minimize immune rejection $[5,6]$ and render them most suitable for stem cell therapy, as evidenced by basic science and clinical studies on their benefits for myocardial healing $[7,8]$. However, the hypoxic-ischemic and inflammatory microenvironments in the ischemic myocardium lowers survival and viability of MSCs [9]. In a previous study, we demonstrated that MSCs transplanted into a porcine myocardial ischemia/reperfusion model were engulfed by cardiac macrophages, which then had a beneficial effect on cardiac function [10]. Moreover, our in vitro study showed that supernatant fluids obtained from macrophages that phagocytized dead MSCs $(\mathrm{pM} \Phi)$ improved survival of hypoxic cardiomyocytes [11]. Although the main mechanisms underlying the therapeutic effects of MSCs remain unknown, $\mathrm{pM} \Phi$ may thus play an important role.

Recent studies have documented the presence of a small amount of cardiac stem cells (CSCs) in the postnatal heart, which have been regarded as one of the most promising stem cell types for myocardial protection and restoration $[12,13]$. The therapeutic effects of CSCs are considered secondary to paracrine mechanisms and cardiovascular lineage differentiation [14,15]. However, it remains undefined how $\mathrm{pM} \Phi$ affect CSCs. Therefore, in the present study, our hypothesis was that whether the supernatant fluids obtained from $\mathrm{pM} \Phi$ could induce CSC differentiation into cardiomyocytes and enhanceanti-hypoxic and proliferative activities. We showed that lipopolysaccharide (LPS)-stimulated macrophages can phagocytize dead MSCs and express more growth and anti-inflammatory factors that are similar to MSCs. Exposure to the supernatants of $\mathrm{pM} \Phi$ enhanced the anti-hypoxia, proliferation, and differentiation properties of CSCs. In the future, some miRNAs or proteins can be extracted from the supernatant fluids that could directly act as a therapy for myocardial infarction. These results give us a new insight into the treatment of myocardial infarction.

\section{Results}

\subsection{Lipopolysaccharide (LPS)-Stimulated Macrophages Phagocytosed Dead Mesenchymal Stem Cells (dMSCs)}

In our previous research, we found that macrophages stimulated by LPS exhibited enhanced phagocytosis of dMSCs [11]. To verify this phenomenon in the present study, dMSCs were measured by flow cytometry (FCM) in three groups. As shown in Figure 1A, the proportion of dMSCs was $0.7 \%$, $31.8 \%$, and $81 \%$ in the no hypoxia, $10 \%$ fetal bovine serum (FBS); $0.5 \% \mathrm{O}_{2}$ for $6 \mathrm{~h}$, phosphate buffered saline (PBS); and $0.5 \% \mathrm{O}_{2}$ for $24 \mathrm{~h}$, PBS groups, respectively. The optimalmultiplicity of infection (MOI) of green fluorescent protein (GFP) transfection was 10.The transfection rate was almost $90 \%$ (not provided). We then co-cultured with or without LPS $(50 \mathrm{ng} / \mathrm{mL})$-stimulated macrophages labeled with PE-anti-F4/ 80 and dMSC (cultured by $0.5 \% \mathrm{O}_{2}$ and PBS for $24 \mathrm{~h}$ ) marked with the FITC-GFP for $48 \mathrm{~h}$, at a cell ratio of 1:2. dMSCs were engulfed by LPS-stimulated macrophages, as was obviously visible under fluorescence microscopy (Figure 1B). The FITC-GFP and PE-anti-F4/80 double-stained cells almost reached $80 \%$ as detected by FCM, and then the double-stained cells were sorted by FCM and defined as "pM $\Phi$ " (Figure 1C,D).

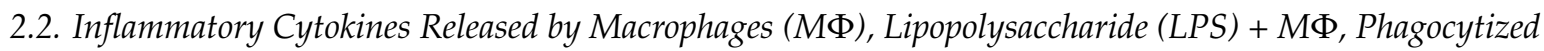 by Macrophages ( $p M \Phi)$}

Inflammatory cytokines secreted by each group were detected by real-time polymerase chain reaction (RT-PCR). Relative expression of the pro-inflammatory cytokines interleukin-6 (IL-6)and tumor necrosis factor- $\alpha$ (TNF- $\alpha$ ) [16] in $\mathrm{pM} \Phi$ was lower than in LPS $+\mathrm{M} \Phi(p<0.001)$, while that of the anti-inflammatory cytokines IL-10 and transforming growth factor- $\beta$ (TGF- $\beta$ ) was higher in $\mathrm{pM} \Phi$ than in LPS $+\mathrm{M} \Phi(p<0.001$, Figure 2). Therefore, these findings show that $\mathrm{pM} \Phi$ appeared to have adopted a more anti-inflammatory phenotype that may be beneficial to injured cells and tissues. 

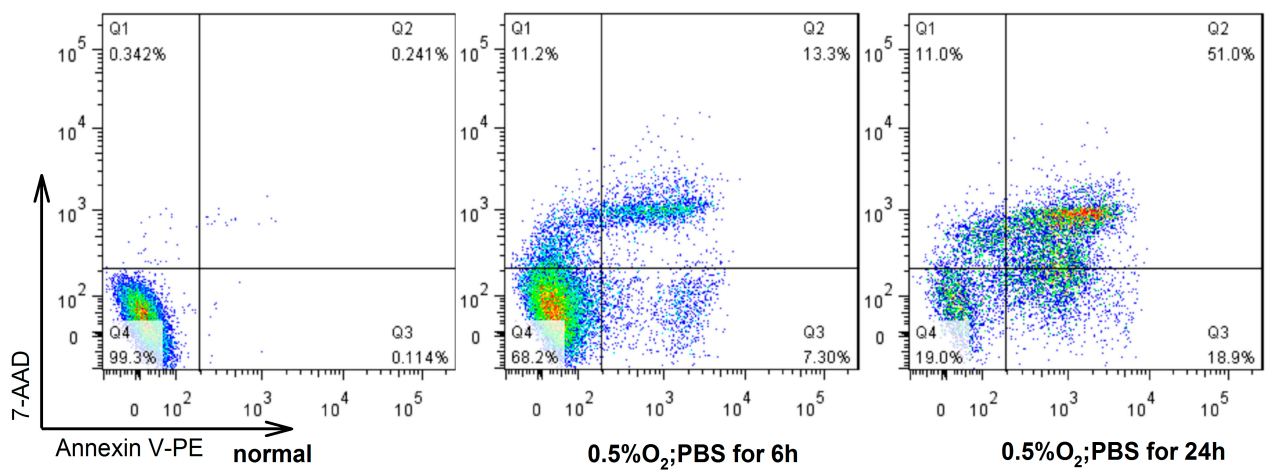

A
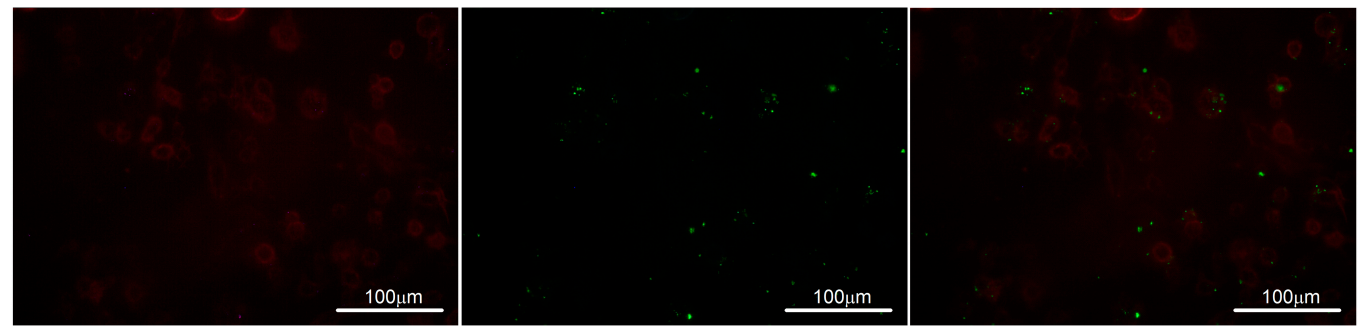

B

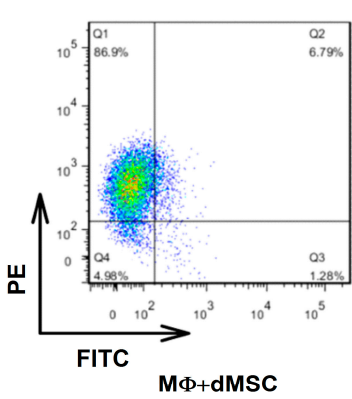

C

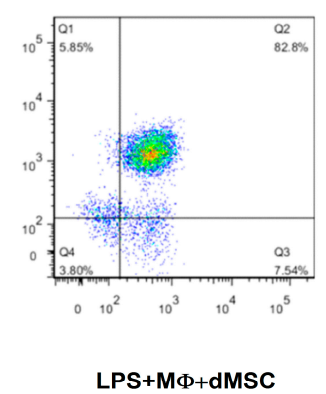

LPS+M $\Phi+d M S C$

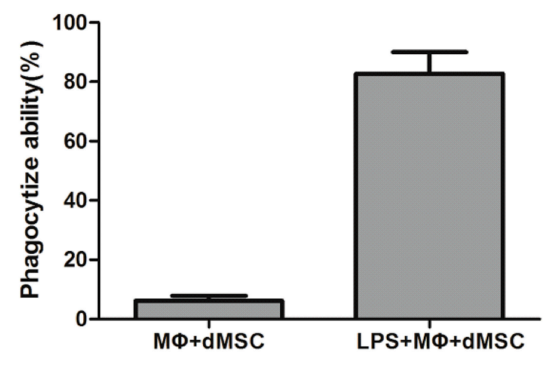

D

Figure 1. Dead mesenchymal stem cells (dMSCs) were phagocytosed by lipopolysaccharide (LPS)-stimulated macrophages. dMSC labeled with FITC-green fluorescent protein (GFP) and activated macrophages (2:1) stained with PE-F4/80 were co-cultured for $48 \mathrm{~h}$, and then detected by fluorescence microscopy and flow cytometry (FCM). (A) MSCs were cultured with three conditions, including no hypoxia and $10 \%$ fetal bovine serum (FBS), $0.5 \% \mathrm{O}_{2}$ for $6 \mathrm{~h}$ and phosphate buffered saline (PBS), $0.5 \%$ $\mathrm{O}_{2}$ for $24 \mathrm{~h}$ and PBS. The ratio of dMSCs was the greatest in third group; (B) Fluorescence microscopy was used to show the phenomenon of phagocytosis; (C,D) Phagocytosis ability of macrophage was evaluated by FCM. Significant difference was shown in two groups with or without LPS $(p<0.001)$, and the mean phagocytosis rate (\%) of LPS-stimulated macrophage was $80 \%$. The percentage of each group and each bar represents the mean value of triplicate results, which means three independent experiments were done. MФ: macrophage.

\subsection{Growth Factors Released by $p M \Phi, M \Phi$ and LPS $+M \Phi$ and Mesenchymal Stem Cells (MSCs)}

RT-PCR was utilized to measure the relative mRNA expression of growth factors in $M \Phi$,

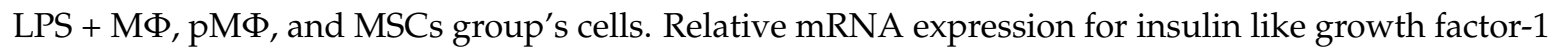
(IGF-1), phenyl glycidyl ether2 (PGE2), keratinocyte growth factor (KGF) and bfibroblast growth actor (bFGF) in $\mathrm{pM} \Phi$ was significantly higher than in $M \Phi$ and LPS + M $\Phi$, and similar or approaching that in MSCs (Figure 3). 
IL-10

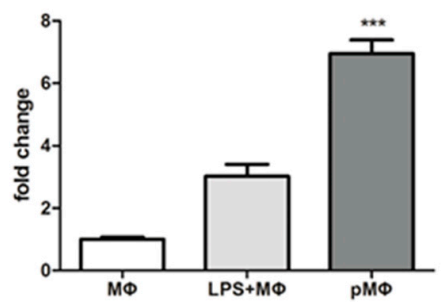

IL-6

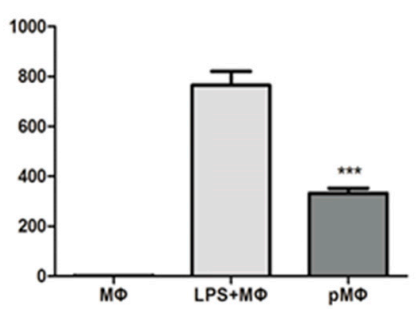

TGF- $\beta$

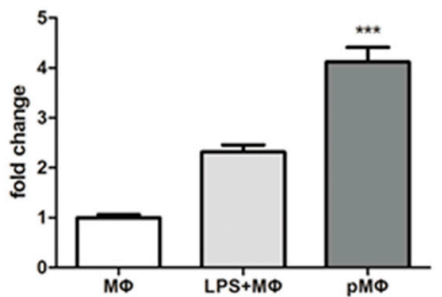

TNF- $\alpha$

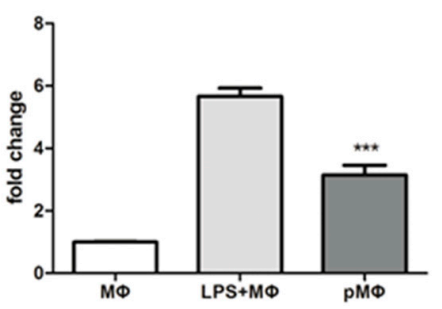

Figure 2. Inflammatory factors secreted by the cell of $M \Phi$, LPS $+M \Phi$, and $\mathrm{pM} \Phi$. Anti-Inflammatory factors interleukin-10 (IL-10) and transforming growth factor- $\beta$ (TGF- $\beta$ ) had higher expression in $\mathrm{pM} \Phi$, and pro-Inflammatory factors IL- 6 and tumor necrosis factor- $\alpha$ (TNF- $\alpha$ ) had lower expression in pM . $^{* * *} p<0.001$ compared with $\mathrm{M} \Phi$. Each bar represents the mean value \pm SD of triplicate results, which means three independent experiments were done.

IGF-1

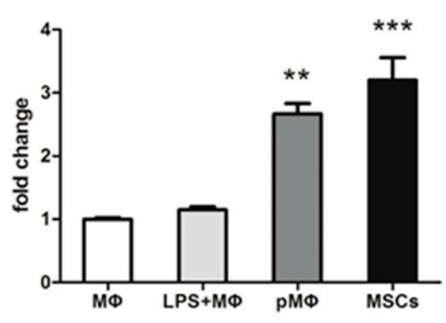

KGF

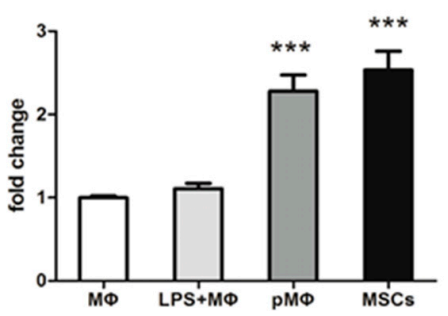

PGE2

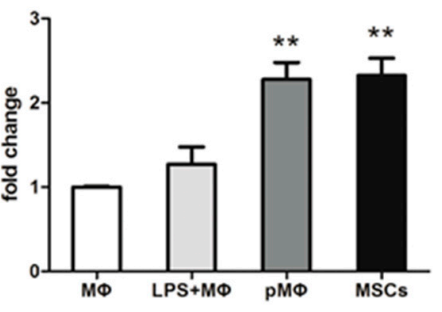

bFGF

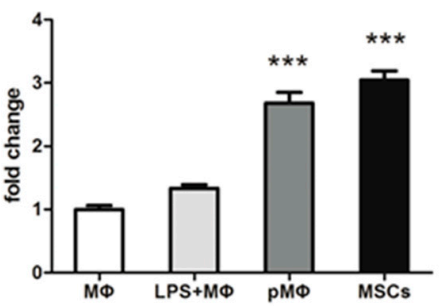

Figure 3. Growth factors were secreted by the cell of M $\Phi$, LPS+ M $\Phi, \mathrm{pM} \Phi$, and MSCs. Compared to $\mathrm{M} \Phi$, all growth factors (Insulin like Growth Factor-1 (IGF-1), Phenyl Glycidyl Ether2 (PGE2), Keratinocyte Growth Factor (KGF), bFibroblast Growth Factor (bFGF)) showed significantly higher expression in $\mathrm{pM} \Phi$, and the results were similar or approaching that of the MSCs $\left({ }^{*} p<0.01\right.$, $\left.{ }^{* * *} p<0.001\right)$. Each bar represents the mean value $\pm S D$ of triplicate results, which means three independent experiments were done.

\subsection{Supernatant Fluids of pMФ Improved Anti-Anoxia and Proliferation of Cardiac Stem Cells (CSCs)}

CSCs were cultured with supernatant fluids which were drawn from M $\Phi$, LPS-M $\Phi$, pM $\Phi$, and MSCs, and then placed in an anoxic environment with a lack of nutrition $\left(0.5 \% \mathrm{O}_{2}, \mathrm{PBS}\right)$ for $0,6,12$, and $24 \mathrm{~h}$. At each time point, cells were collected and apoptosis was analyzed by FCM. The anti-anoxia 
status of CSCs was significantly enhanced at 6,12 , and $24 \mathrm{~h}$ by the presence of supernatant fluids from pM $\Phi$ (Figure 4A,B). CSCs proliferation assessed using cell counting kit-8 (CCK-8)—was increased in all groups (Figure $4 \mathrm{C}$ ), but was significantly higher in $\mathrm{PM} \Phi$ and MSCs than in M $\Phi$ at 6, 12, and $24 \mathrm{~h}$.

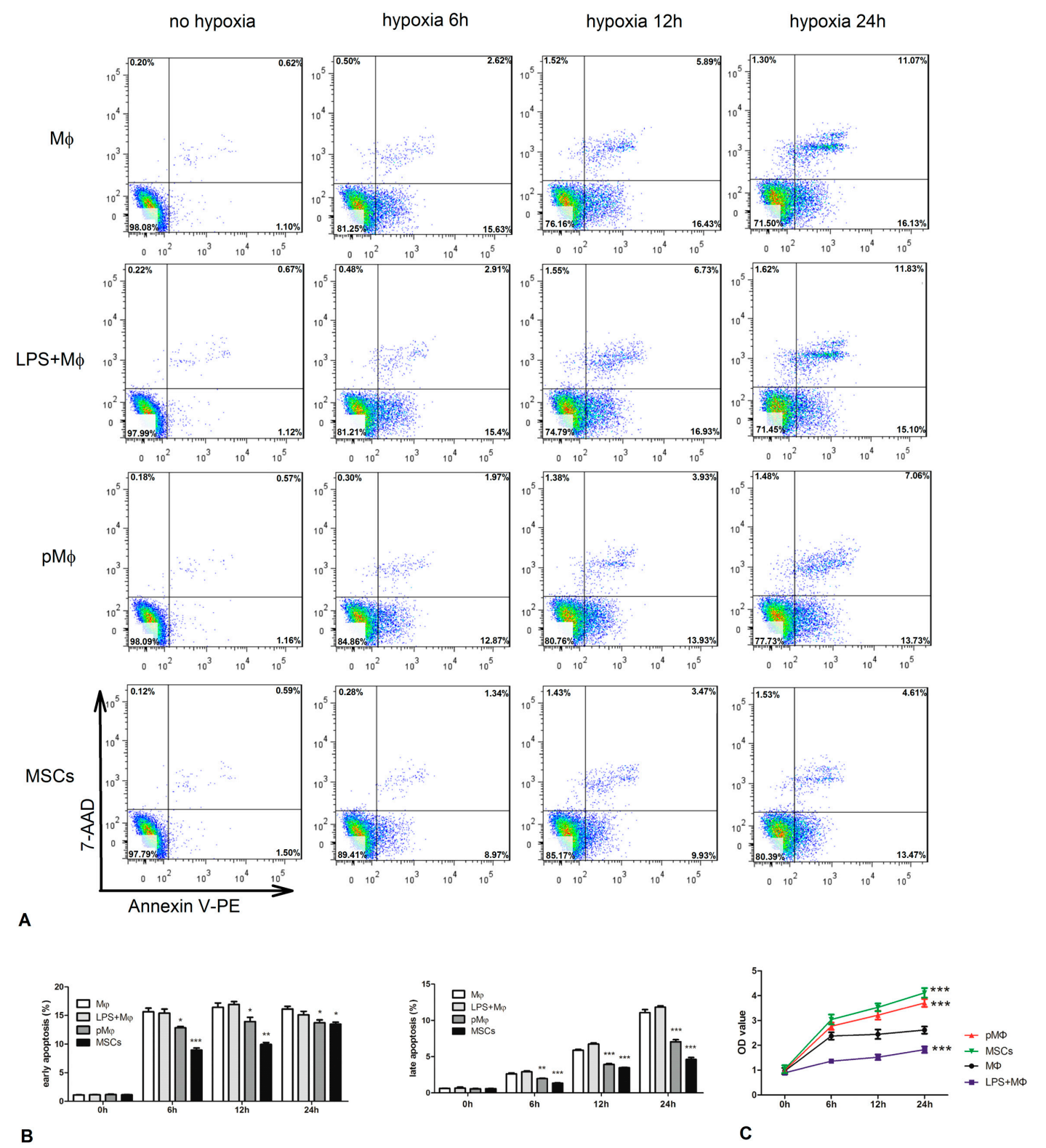

Figure 4. Cell counting kit-8 (CCK-8) proliferation assay and Annexin V-PE/7-ADD detected by FCM were used to measure the proliferation and anti-hypoxic properties of CSCs. (A,B) CSCs which were co-cultured with the supernatants of M $\Phi, \mathrm{LPS}-\mathrm{M} \Phi, \mathrm{pM} \Phi$, and MSCs were harvested in 0, 6, 12, and $24 \mathrm{~h}$ in hypoxia $\left(0.5 \% \mathrm{O}_{2}\right)$ conditions. Compared to the M $\Phi$ and LPS-M $\Phi$, the proportion of apoptosis in CSCs was significantly lower in $\mathrm{pM} \Phi$ and MSCs at each time point; (C) The proliferation of CSCs detected by CСK-8 was dramatically increased in $\mathrm{pM} \Phi$ and MSCs groups. ${ }^{*} p<0.05 ;{ }^{* *} p<0.01$; and $* * *<0.001$ compared with $\mathrm{M} \Phi$. The percentage of each group represents the mean value of triplicate results, which means three independent experiments were done.

\subsection{Supernatant Fluids of $p M \Phi$ Induced CSCs Differentiation}

CSCs markers were identified by FCM. After isolation, CSCs had high expression of GATA-4 (38\%) and low expression of TnI ( $0.375 \%$, Figure 5A). The CSCs were cultured for 7 days in the presence 
of supernatant fluids from M $\Phi$, LPS-M $\Phi$, pM $\Phi$, and MSCs. GATA-4 and TnI were significantly higher in $\mathrm{PM} \Phi$ than $M \Phi$ and LPS-M $\Phi$, with similar levels between $\mathrm{pM} \Phi$ and MSCs (Figure $5 \mathrm{~B}, \mathrm{C}$ ). Seven days later GATA-4 and TnI of CSCs in the presence of the supernatant fluids of $\mathrm{pM} \Phi$ almost attained $5.69 \%$ and $72.1 \%$, which was significantly higher than M $\Phi$ and LPS-M $\Phi$, and similar to the levels of the MSC group (Figure 5B,C).

A
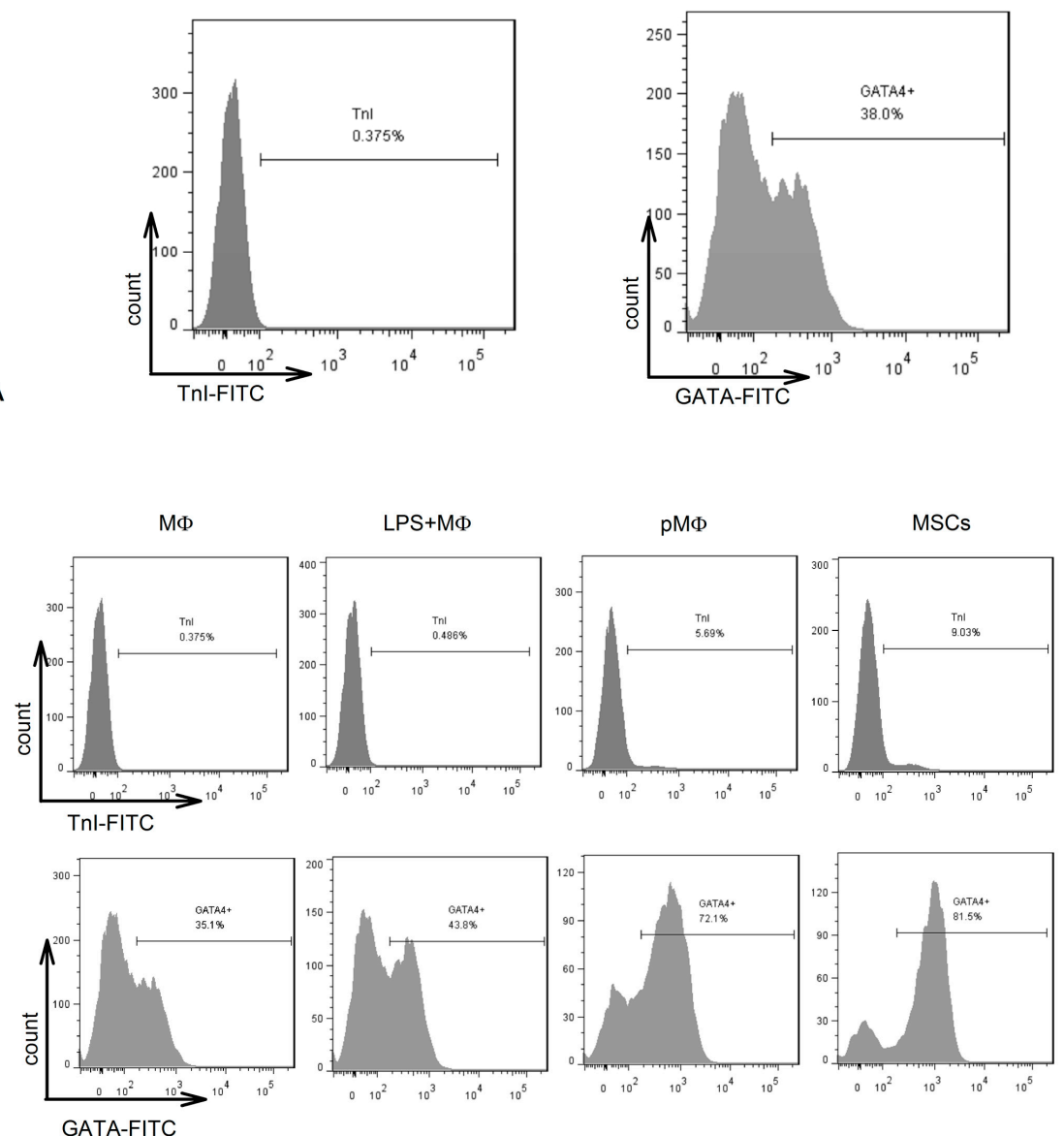

B
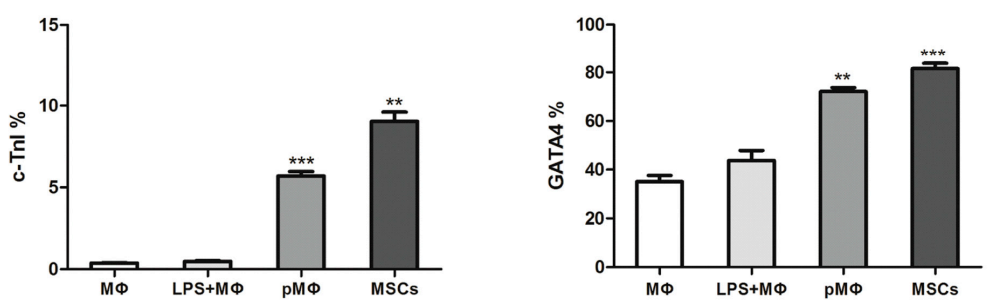

Figure 5. The markers of cardiomyocytes were detected in CSCs. (A) CSCs were obtained from the hearts of mice and then GATA-4 and TnI were detected by FCM; $(\mathbf{B}, \mathbf{C})$ CSCs co-cultured with the supernatants of M $\Phi$, LPS-M $\Phi$, $\mathrm{pM} \Phi$, and MSCs were harvested after 7 days. FCM was used to detect cardiomyocytes markers. GATA-4 and TnI showed significantly higher expression in the $\mathrm{pM} \Phi$ and MSC groups; ${ }^{* *} p<0.01$; and ${ }^{* * *} p<0.001$, compared with $M \Phi$. The percentage and the bar of each group represent the mean value of triplicate results, which means that three independent experiments were done.

\section{Discussion}

In the present study, LPS-stimulated macrophages phagocytized dMSCs (pM $\Phi$ ) and then expressed more growth and anti-inflammatory factors and less pro-inflammatory factors. 
CSCs cultured in the presence of the supernatant fluids of these $\mathrm{pM} \Phi$ not only exhibited enhanced proliferative and anti-hypoxic activities, but also differentiation. To our knowledge, this study is the first to document the beneficial effects of $\mathrm{pM} \Phi$ on CSCs, which might constitute one of the mechanisms underlying the persistence of therapeutic effects of dMSCs despite their massive demise after transplantation into myocardial infarction (MI) models.

Although the specific mechanisms whereby MSCs promote improved cardiac function has been undefined, it is known that most MSCs die shortly after implantation because of the unwelcoming microenvironments of MI [17-19], and that apoptotic cells are phagocytized by activated macrophages $[20,21]$. Phagocytosis of dMSCs by activated macrophages could mediate the observed effects of MSCs after their demise. In a previous study, MSCs that overexpressed the heme oxygenase-1 (HO-1) gene and stained with super paramagnetic iron oxide (SPIO) significantly improved cardiac function compared with other MSCs after transplantation into a porcine myocardial ischemia/reperfusion model. However, one week later, signal voids caused by the SPIO were observed by MRI in the surrounding area of myocardial infarction, reflecting that cardiac macrophages had phagocytized SPIO-labeled MSCs, as shown by histological analysis [10]. In our study, we found that activated macrophages induced by LPS phagocytized dMSCs, which was consistent with the aforementioned in vivo and in vitro studies $[10,11]$. We could thus infer that, compared to normal macrophages, $\mathrm{pM} \Phi$ may have changed and played a similar function to MSCs on the repair of the injured heart.

Activated macrophages can be divided into M1 and M2 macrophages [16,22,23]. M1 phenotype macrophages (classically activated macrophages) mount inflammatory reactions by secreting many pro-inflammatory cytokines, such as IL-1 $\beta$ and TNF- $\alpha$, among others [16], while M2 phenotype macrophages (alternatively activated macrophages) secrete many anti-inflammatory cytokines such as IL-10, Arg-1, and TGF- $\beta$, involved in anti-inflammatory reactions and tissue remodeling [16]. In the present study, we found that compared with LPS-M $\Phi$, pM $\Phi$ exhibited upregulated IL-10 and TGF- $\beta$ expression and downregulated IL-1 and TNF- $\alpha$ expression, which might reflect a $\mathrm{pM} \Phi$-induced macrophage polarization toward an M2 phenotype compatible with tissue repair, and with the observed enhanced anti-hypoxic and anti-apoptotic activities of CSCs similar to those of MSCs at different time points.

Another important finding of the present study was that expression of GATA-4 (72.1\%) and c-TnI (5.69\%) by CSCs as detected by FCM was significantly increased following incubation with the supernatant fluids of $\mathrm{pM} \Phi$ for 7 days to a similar extent as that of MSCs. c-TnI, one of troponin subunits, regulates $\mathrm{Ca}^{2+}$-activated tension in cardiac and striated muscle, but not smooth muscle [24-26]. The function of c-TnI is to inhibit tension development in striated muscle until $\mathrm{Ca}^{2+}$ binds to another troponin subunit on troponin $\mathrm{C}(\mathrm{TnC})$ [24]. c-TnI is considered a unique marker of myocardial tissue [25-28], and greater differentiation into the cardiomyocyte phenotype is reflected in higher cytoplasmic c-TnI expression. Among the GATA family of zinc finger transcription factors, GATA-4 is expressed in the heart [29]. Knockdown of GATA-4 using an antisense strategy can terminate the differentiation of cardiac progenitor cells (CPC) and induce CPC apoptosis, while its overexpression can lead to ectopic beating cardiomyocytes and even an hypertrophic response [29,30]. Thus, GATA-4 is a mediator of CPC differentiation, plays an important role in progenitor cell proliferation, and is another cardiomyocyte marker. The aforementioned results also indicate that the supernatant fluids of pM $\Phi$ induced CSC differentiation into cardiomyocytes and enhanced proliferation of CSCs to a similar extent as MSCs.

In contrast to pro-inflammatory factors, levels of growth factors such as IGF-1, VEGF- $\alpha$, KGF, and bFGF secreted by the $\mathrm{pM} \Phi$ group were higher than those of M $\Phi$ and LPS-M $\Phi$, and similar to those of MSCs, which is consistent with findings in our previous report [11]. Growth factors are recognized to have anti-apoptotic effects and the capacity to induce cell differentiation [31-33]. For example, overexpression of IGF-1 in CSCs enhances the repair of injured cardiac tissue [32] and promotes CSCs differentiation, while VEGF- $\alpha$ replenishes cardiomyocytes by inducing pluripotent 
stem cell differentiation [33]. Therefore, the induction of CSC differentiation and proliferation by the supernatant fluids of $\mathrm{pM} \Phi$ may be related to these growth factors. However, the specific mechanisms of this phenomenon were not studied here. Some other key factors, such as miRNA or proteins in the supernatant fluids, need to be found, not just anti-inflammation and growth cytokine. Furthermore, animal experiments and clinical research are warranted to verify the observations and specific mechanisms presented.

The findings of the present study provide new insight into the therapeutic effect of MSCs after transplantation into a myocardial infarction model, and serve as the basis for future research into the specific mechanisms of macrophage phenotype change, CSC differentiation, and the various cytokines secreted by $\mathrm{pM} \Phi$. Further animal experiments and clinical research are warranted to verify the observations presented.

\section{Materials and Methods}

\subsection{Animals}

C57BL/6 mice (6 to 10 weeks old) were purchased from Slac Laboratory Animal Co., Ltd. (Shanghai, China) and kept under specific pathogen-free conditions. All animal experiments were approved by the Ethics Committee of Xinhua Hospital affiliated with Shanghai Jiaotong University School of Medicine, Shanghai China and the approval No. was XHEC-F-2016-003.

\subsection{Cell Isolation and Culture}

Seven-to-10 week-old male C57BL/ 6 mice were used to isolate bone marrow-derived MSCs. The mice were anesthetized by using pentobarbital $(50 \mathrm{mg} / \mathrm{kg}$, intraperitoneal injection) to minimize suffering and killed by cervical dislocation without recovery from anesthesia. Tibias and femurs then were removed in sterile conditions. The bone ends were cut and the bone marrow was flushed using PBS (Gibco, Carlsbad, CA, USA, Cat\#8115178) supplemented with 1\%-2\% Fetal Bovine Serum (FBS, Gibco, Cat\#10099-141). The cell suspension was then filtered through a $40 \mu \mathrm{m}$ strainer (BD Falcon, Franklin Lakes, NJ, USA, Cat\#352340). After centrifugation of the filtered suspension at $1000 \mathrm{rpm} / \mathrm{min}$ for $5 \mathrm{~min}$, the bone marrow cells were cultured in Dulbecco's Modified Eagle Medium: Nutrient Mixture F-12 (DMEM/F12, HyClone, Beijing, China, Cat\#SH30023.FS) supplemented with 10\% FBS and $1 \%$ penicillin-streptomycin (Gibco, Cat\#C0222). The culture medium was changed on the fifth day to remove non-adherent cells, and the cells were passaged two days later. MSCs were passaged by using Trypsin (0.25\%) including Ethylene Diamine Tetraacetic Acid (EDTA) (Gibco, Cat\#25200-056) and identified by flow cytometry (FCM, BD Biosciences, San Jose, CA, USA). The ensuing experiments used passage three cells.

Six-to-8 week-old male C57BL/ 6 mice were used to isolate peritoneal macrophages. The mice were injected with $1 \mathrm{~mL}$ thioglycolate (4\%, Sigma-Aldrich, St. Louis, MO, USA, Cat\#B2551) and sacrificed 4 days later. After using scissors to cut open the skin over the abdominal area without puncturing the peritoneum, a $10 \mathrm{~mL}$ syringe with an $18 \mathrm{G}$ needle was used to inject $10 \mathrm{~mL}$ Roswell Park Memorial Institute-1640 (RPMI-1640 HyClone, Cat\#SH30255.FS) with 10\% FBS into the abdominal cavity. The belly then was pressed about 20 times, and media was withdrawn using a syringe and subsequently injected into six well plates and incubated at $37^{\circ} \mathrm{C}$ for $3-4 \mathrm{~h}$. Supernatant fluid was aspirated and the cells were washed thrice with PBS before being incubated overnight. Cells were collected on the following day and labeled with PE-anti-F4/80 (eBioscience, Carlsbad, CA, USA, Cat\#12-4801-80) to identify peritoneal macrophages by FCM.

Cardiac stem cells were obtained from the hearts of 7-8 week-old male C57BL/ 6 mice via a two-step protocol, as previously described [34,35]. Firstly, the hearts of mice were chopped and cultured for 2-3 weeks until the small, round, phase-bright cells migrated from the adherent heart tissue and proliferated over a fibroblast layer. Then, the small, round, phase-bright cells were isolated through magnetic-activated cell sorting (MACS) with Sca-1 magnetic beads (MiltenyiBiotec Inc., Auburn, CA, 
USA) to obtain Sca- 1 + cells as instructed by the manufacturers' protocols. The selected Sca- 1 cells were cultured and maintained in ISCOVE'S modified DMEM (IMDM, HyClone, Cat\#SH30228.FS) supplemented with 20\% FBS, $1 \%$ penicillin-streptomycin, 1\% L-glutamine (Sigma-Aldrich, Cat\#59202C) and $0.0068 \mu \mathrm{L} / \mathrm{mL} \beta$-mercaptoethanol (Sigma-Aldrich, Cat\# 07604).

\subsection{GFP Transfection}

A mouse lentivirus carrying GFP reporter genes (Bio-Link, SHH, Shanghai, China) was transfected into MSCs at passage 4. MSCs were seeded in a six-well plate at $1 \times 10^{5}$ cells per well, grown overnight. Next day, cell culture was aspirated and $1 \mathrm{~mL}$ cell medium without penicillin-streptomycin was added into MSCs which contain $5 \mu \mathrm{g} / \mathrm{mL}$ polybrene and lentivirus at a MOI of 1,5 , and 10 . After transfection for $12 \mathrm{~h}$, virus-containing supernatant was aspirated and replaced with complete culture medium. Fluorescence microscopy was used to observe transfection efficiency, and transfection rate was evaluated by FCM after $48 \mathrm{~h}$. The optimal MOI was chosen for highest GFP expression.

\subsection{Detection of MSC Apoptosis and Isolation of $p M \Phi$}

The three groups in this experiment were: normal; $0.5 \% \mathrm{O}_{2}$ for $6 \mathrm{~h}$ and cultured with PBS; and $0.5 \% \mathrm{O}_{2}$ for $24 \mathrm{~h}$ and cultured with PBS. Then, dMSCs in these three groups were measured by FCM. Annexin V-PE/7-AAD (eBioscience, Cat\#559763) double staining was used to identify the apoptosis rate of MSCs. The cells $\left(2 \times 10^{6}\right.$ cells $\left./ \mathrm{mL}\right)$ were harvested, washed twice with ice-cold PBS, and incubated for $15 \mathrm{~min}$ in 1X Annexin V Binding Buffer containing 7-AAD-Percp and Annexin V-PE. Finally, apoptosis was detected by FCM and analyzed by the FlowJo software (Tree Star Inc., Ashland, OR, USA). Experiments were carried out in triplicate.

dMSCs of the third group were plated in six-well plates, and macrophages were immediately added to each well with or without of LPS (50 ng/mL, Sigma-Aldrich, Cat\#L2630). Co-cultured cells were incubated for another $48 \mathrm{~h}$, and non-adherent cells (i.e., dMSCs that were not phagocytized by macrophages) were removed. According to FITC-GFP and PE-anti-F4/80, the adherent cells were sorted by FCM to obtain $\mathrm{PM} \Phi$, which then were incubated for an additional period of $24 \mathrm{~h}$ to obtain the supernatant fluids. Supernatant fluids were collected and stored at $-20{ }^{\circ} \mathrm{C}$ until use, and cells were collected into a centrifuge tube for real-time PCR. Phagocytosis was evaluated by fluorescence microscope (Leica, Wetzlar, Germany).

\subsection{Cell Viability Assay}

CCK-8 assay was used to measure the viability of CSCs. CSCs (1000/well) were seeded in 96-well plates overnight and then incubated with the supernatant fluids of M $\Phi$, LPS + M $\Phi, \mathrm{pM} \Phi$, and MSCs. Each group was tested in triplicate in three replicate wells. Ten $\mu \mathrm{L}$ CCK-8 solution (DOJINDO, Kumamoto, Japan, Cat\#AY-4710P) was added into the cells of the four groups at 0, 6, 12, and $24 \mathrm{~h}$, respectively. After incubation for another $4 \mathrm{~h}$, the optical density (OD) values were determined at $450 \mathrm{~nm}$ by using a microplate reader (BioTek, Winooski, VT, USA) at each time point.

\subsection{Real-Time PCR Analysis}

Expression of IGF-1, VEGF-A, KGF, bFGF, IL-10, TGF- $\beta$, IL-6, and TNF- $\alpha$ in M $\Phi$, LPS + M $\Phi$, pM $\Phi$, and MSC cells was tested by real-time PCR Analysis. Total RNA was obtained from the cells in the four groups using TRIzol reagent (Takara, Dalian, China, Cat\#RR036A) following the manufacturer's instructions, and $1 \mu \mathrm{g}$ of total RNA was reverse-transcribed to cDNA using the PrimeScript RT Master Mix kit (Takara) according to the manufacturer's instructions. The SYBR Premix Ex TaqTM kit (Takara, Cat\# RR420A) in a total volume of 20 with $2 \mu \mathrm{L}$ of cDNA primers $(0.2 \mathrm{mM}$ each), $10 \mu \mathrm{L}$ of SYBR Green, and $0.4 \mu \mathrm{L}$ Rox Dye II was used to perform the real-time PCR Analysis. The samples were run on an ABI 7500 detector (Applied Biosystems, Foster City, CA, USA) with standard PCR conditions: $95^{\circ} \mathrm{C}$ for $30 \mathrm{~s}$, followed by 40 cycles of $95^{\circ} \mathrm{C}$ for $5 \mathrm{~s}$ and $60^{\circ} \mathrm{C}$ for $34 \mathrm{~s}$, with a final dissociation stage. GAPDH cDNA (Sangon Biotech, Shanghai, China) was used as endogenous control to normalize the 
$\mathrm{Ct}$ value for target genes. The sequences of the primers (Sangon Biotech) for the target genes are listed in Table 1.

Table 1. Sequences of forward and reverse primers for target genes.

\begin{tabular}{ll}
\hline Target Gene & Forward and Reverse of Prime \\
\hline$I L-10$ & 5'-TGCACTACCAAAGCCACAAG-3'; 5'-TGATCCTCATGCCAGTCAGT-3' \\
Il-6 & 5'-TCTGCAAGAGACTTCCATCCA-3'; 5'-AGTCTCCTCTCCGGACTTGT-3' \\
$T N F-\alpha$ & 5'-GGTGCCTATGTCTCAGCCTC-3'; 5'-CCACTTGGTGGTTTGGAGTG-3' \\
TGF- $\beta$ & 5'-ATTCCTGGCGTTACCTTGG-3'; 5'-AGCCCTGTATTCCGTCTCCT \\
$I G F-1$ & 5'-GGGCTGAGCTGGTGGATG-3'; 5'-CTCCAGTCTCCTCAGATCAC-3' \\
$P G E 2$ & 5'-GGAGACTCTTCGAGGAGCACTT-3'; 5'-GGCGATTTAGCAGCAGATATAAGAA-3' \\
KGF & 5'-CTGCCAACTCTGCTCTACAG-3'; 5'-TCCAACTGCCACGGTCCTGAT-3' \\
$b F G F$ & 5'-AAGGGAGTGTGTGCCAACC-3'; 5'-GCCCAGTTCGTTTCAGTGC-3' \\
\hline
\end{tabular}

\subsection{Flow Cytometry (FCM) Analysis}

Anti-hypoxic activity and CSC markers were detected by FCM Analysis. CSCs were incubated with the supernatant fluids of M $\Phi$, LPS + M $\Phi, \mathrm{pM} \Phi$, and MSCs. Cells were then harvested after $0,6,12$, and $24 \mathrm{~h}$ in hypoxia $\left(0.5 \% \mathrm{O}_{2}\right)$, respectively. Apoptosis of CSCs was determined using the Annexin V-PE/7-ADD as previously described. Three steps were followed to determine CSC markers. In step one, the cells were fixed with $4 \%$ paraformaldehyde (Fushen, Shanghai, China, Cat\#FS030101) for $10 \mathrm{~min}$ and then permeabilized with $0.1 \%$ PBS-Tween20 (Beyotime, Shanghai, China, Cat\#ST825) for $20 \mathrm{~min}$. In step two, the cells were washed thrice with PBS and resuspended with $100 \mu \mathrm{L}$ PBS, and then primary antibody was added for $30 \mathrm{~min}$ at room temperature. The secondary antibody was added for $30 \mathrm{~min}$ at room temperature, and the cells were washed thrice and resuspended with $100 \mu \mathrm{L}$ PBS. Then, the cells were washed and resuspended in PBS and identified by FCM; the results were analyzed using FlowJo software. The primary antibody against GATA-4 (Cat\#ab134057) and TnI (Cat\#ab47003) and the secondary antibodyAlexa Fluor ${ }^{\circledR} 488$ goat anti-rabbit IgG $(\mathrm{H}+\mathrm{L})$ (Cat\#ab150077) were purchased from Abcam (Cambridge, UK), and used at the recommended concentrations in experiments carried out in triplicate.

\subsection{Statistical Analysis}

Statistical analysis was performed using SPSS software 19.0 (SPSS Inc., Chicago, IL, USA). All values are expressed as mean $\pm \mathrm{SD}$. One-way analysis of variance (ANOVA) was used to assess the effects of one factor among multiple groups, and a Tukey test was used for post hoc testing. Statistical significance was defined as $p<0.05$.

\section{Conclusions}

Activated macrophages that had phagocytized dMSCs (termed $\mathrm{pM} \Phi$ ) expressed higher levels of anti-inflammatory cytokines, lower levels of pro-inflammatory cytokines, and secreted many growth factors. CSCs incubated with the supernatant fluids of said pM $\Phi$ exhibited enhanced anti-hypoxic and proliferative activities and differentiation into cardiomyocytes. The latter findings provide us a new understanding about the mechanisms underlying the therapeutic effect of MSCs after transplantation into a myocardial infarction model. Future study should focus on the extraction of some miRNAs or proteins from the supernatant fluids that could directly act as a therapy for myocardial infarction. These results provide us with a new horizon for the treatment of myocardial infarction.

Acknowledgments: This work was supported by Nature Science Foundation of China Grant 81170193, 81070084 and Shanghai Medical Key Specialty Construction Projects (Class A, series number: ZK2012A24).

Author Contributions: Chengxing Shen and Liang Liu conceived and designed the experiments; Liang Liu and Xian Jin performed the experiments; Xian Jin and Zhong'e Zhou analyzed the data; Chengxing Shen contributed 
reagents and materials; Liang Liu, Xian Jin and Chengxing Shen wrote the paper. All authors approved its final version and agree to be accountable for all aspects of the work.

Conflicts of Interest: The authors declare no conflict of interest.

\section{References}

1. Gaziano, T.A. Cardiovascular disease in the developing world and its cost-effective management. Circulation 2005, 112, 3547-3553. [CrossRef] [PubMed]

2. Segers, V.F.; Lee, R.T. Stem-cell therapy for cardiac disease. Nature 2008, 451, 937-942. [CrossRef] [PubMed]

3. Schachinger, V.; Assmus, B.; Britten, M.B.; Honold, J.; Lehmann, R.; Teupe, C.; Abolmaali, N.D.; Vogl, T.J.; Hofmann, W.K.; Martin, H.; et al. Transplantation of progenitor cells and regeneration enhancement in acute myocardial infarction: Final one-year results of the topcare-ami trial. J. Am. Coll. Cardiol. 2004, 44, 1690-1699. [CrossRef] [PubMed]

4. Pittenger, M.F.; Mackay, A.M.; Beck, S.C.; Jaiswal, R.K.; Douglas, R.; Mosca, J.D.; Moorman, M.A.; Simonetti, D.W.; Craig, S.; Marshak, D.R. Multilineage potential of adult human mesenchymal stem cells. Science 1999, 284, 143-147. [CrossRef] [PubMed]

5. Pittenger, M.F.; Martin, B.J. Mesenchymal stem cells and their potential as cardiac therapeutics. Circ. Res. 2004, 95, 9-20. [CrossRef] [PubMed]

6. Hass, R.; Kasper, C.; Bohm, S.; Jacobs, R. Different populations and sources of human mesenchymal stem cells (MSC): A comparison of adult and neonatal tissue-derived MSC. Cell Commun. Signal. 2011, 9, 12. [CrossRef] [PubMed]

7. Miyahara, Y.; Nagaya, N.; Kataoka, M.; Yanagawa, B.; Tanaka, K.; Hao, H.; Ishino, K.; Ishida, H.; Shimizu, T.; Kangawa, K.; et al. Monolayered mesenchymal stem cells repair scarred myocardium after myocardial infarction. Nat. Med. 2006, 12, 459-465. [CrossRef] [PubMed]

8. Price, M.J.; Chou, C.C.; Frantzen, M.; Miyamoto, T.; Kar, S.; Lee, S.; Shah, P.K.; Martin, B.J.; Lill, M.; Forrester, J.S.; et al. Intravenous mesenchymal stem cell therapy early after reperfused acute myocardial infarction improves left ventricular function and alters electrophysiologic properties. Int. J. Cardiol. 2006, 111, 231-239. [CrossRef] [PubMed]

9. Li, Q.; Wang, Y.; Deng, Z. Pre-conditioned mesenchymal stem cells: A better way for cell-based therapy. Stem Cell Res. Ther. 2013, 4, 63. [CrossRef] [PubMed]

10. Jiang, Y.B.; Chen, L.J.; Tang, Y.L.; Ma, G.S.; Shen, C.X.; Qi, C.M.; Zhu, Q.; Yao, Y.Y.; Liu, N.F. Ho-1 gene overexpression enhances the beneficial effects of superparamagnetic iron oxide labeled bone marrow stromal cells transplantation in swine hearts underwent ischemia/reperfusion: An MRI study. Basic Res. Cardiol. 2010, 105, 431-442. [CrossRef] [PubMed]

11. Lu, W.; Fu, C.; Song, L.; Yao, Y.; Zhang, X.; Chen, Z.; Li, Y.; Ma, G.; Shen, C. Exposure to supernatants of macrophages that phagocytized dead mesenchymal stem cells improves hypoxic cardiomyocytes survival. Int. J. Cardiol. 2013, 165, 333-340. [CrossRef] [PubMed]

12. Laugwitz, K.L.; Moretti, A.; Lam, J.; Gruber, P.; Chen, Y.; Woodard, S.; Lin, L.Z.; Cai, C.L.; Lu, M.M.; Reth, M.; et al. Postnatal isl1 + cardioblasts enter fully differentiated cardiomyocyte lineages. Nature 2005, 433, 647-653. [CrossRef] [PubMed]

13. Oh, H.; Bradfute, S.B.; Gallardo, T.D.; Nakamura, T.; Gaussin, V.; Mishina, Y.; Pocius, J.; Michael, L.H.; Behringer, R.R.; Garry, D.J.; et al. Cardiac progenitor cells from adult myocardium: Homing, differentiation, and fusion after infarction. Proc. Natl. Acad. Sci. USA 2003, 100, 12313-12318. [CrossRef] [PubMed]

14. Urbich, C.; Aicher, A.; Heeschen, C.; Dernbach, E.; Hofmann, W.K.; Zeiher, A.M.; Dimmeler, S. Soluble factors released by endothelial progenitor cells promote migration of endothelial cells and cardiac resident progenitor cells. J. Mol. Cell. Cardiol. 2005, 39, 733-742. [CrossRef] [PubMed]

15. Tang, X.L.; Rokosh, G.; Sanganalmath, S.K.; Yuan, F.; Sato, H.; Mu, J.; Dai, S.; Li, C.; Chen, N.; Peng, Y.; et al. Intracoronary administration of cardiac progenitor cells alleviates left ventricular dysfunction in rats with a 30-day-old infarction. Circulation 2010, 121, 293-305. [CrossRef] [PubMed]

16. Italiani, P.; Boraschi, D. From monocytes to M1/M2 macrophages: Phenotypical vs. Functional differentiation. Front. Immunol. 2014, 5, 514. [CrossRef] [PubMed]

17. Janssens, S.; Dubois, C.; Bogaert, J.; Theunissen, K.; Deroose, C.; Desmet, W.; Kalantzi, M.; Herbots, L.; Sinnaeve, P.; Dens, J.; et al. Autologous bone marrow-derived stem-cell transfer in patients with ST-segment 
elevation myocardial infarction: Double-blind, randomised controlled trial. Lancet 2006, 367, 113-121. [CrossRef]

18. Barbash, I.M.; Chouraqui, P.; Baron, J.; Feinberg, M.S.; Etzion, S.; Tessone, A.; Miller, L.; Guetta, E.; Zipori, D.; Kedes, L.H.; et al. Systemic delivery of bone marrow-derived mesenchymal stem cells to the infarcted myocardium: Feasibility, cell migration, and body distribution. Circulation 2003, 108, 863-868. [CrossRef] [PubMed]

19. Winkler, T.; von Roth, P.; Schuman, M.R.; Sieland, K.; Stoltenburg-Didinger, G.; Taupitz, M.; Perka, C.; Duda, G.N.; Matziolis, G. In vivo visualization of locally transplanted mesenchymal stem cells in the severely injured muscle in rats. Tissue Eng. A 2008, 14, 1149-1160. [CrossRef] [PubMed]

20. Lichtenauer, M.; Mildner, M.; Baumgartner, A.; Hasun, M.; Werba, G.; Beer, L.; Altmann, P.; Roth, G.; Gyongyosi, M.; Podesser, B.K.; et al. Intravenous and intramyocardial injection of apoptotic white blood cell suspensions prevents ventricular remodelling by increasing elastin expression in cardiac scar tissue after myocardial infarction. Basic Res. Cardiol. 2011, 106, 645-655. [CrossRef] [PubMed]

21. Paidassi, H.; Tacnet-Delorme, P.; Arlaud, G.J.; Frachet, P. How phagocytes track down and respond to apoptotic cells. Crit. Rev. Immunol. 2009, 29, 111-130. [CrossRef] [PubMed]

22. Liu, Y.C.; Zou, X.B.; Chai, Y.F.; Yao, Y.M. Macrophage polarization in inflammatory diseases. Int. J. Biol. Sci. 2014, 10, 520-529. [CrossRef] [PubMed]

23. Labonte, A.C.; Tosello-Trampont, A.C.; Hahn, Y.S. The role of macrophage polarization in infectious and inflammatory diseases. Mol. Cells 2014, 37, 275-285. [CrossRef] [PubMed]

24. Tobacman, L.S. Thin filament-mediated regulation of cardiac contraction. Annu. Rev. Physiol. 1996, 58, 447-481. [CrossRef] [PubMed]

25. Bodor, G.S.; Survant, L.; Voss, E.M.; Smith, S.; Porterfield, D.; Apple, F.S. Cardiac troponin t composition in normal and regenerating human skeletal muscle. Clin. Chem. 1997, 43, 476-484. [PubMed]

26. Skeik, N.; Patel, D.C. A review of troponins in ischemic heart disease and other conditions. Int. J. Angiol. 2007, 16, 53-58. [CrossRef] [PubMed]

27. Clause, K.C.; Tchao, J.; Powell, M.C.; Liu, L.J.; Huard, J.; Keller, B.B.; Tobita, K. Developing cardiac and skeletal muscle share fast-skeletal myosin heavy chain and cardiac troponin-I expression. PLoS ONE 2012, 7, e40725. [CrossRef] [PubMed]

28. Adams, J.E., 3rd; Bodor, G.S.; Davila-Roman, V.G.; Delmez, J.A.; Apple, F.S.; Ladenson, J.H.; Jaffe, A.S. Cardiac troponin I. A marker with high specificity for cardiac injury. Circulation 1993, 88, 101-106. [CrossRef] [PubMed]

29. Suzuki, Y.J.; Evans, T. Regulation of cardiac myocyte apoptosis by the GATA-4 transcription factor. Life Sci. 2004, 74, 1829-1838. [CrossRef] [PubMed]

30. Peterkin, T.; Gibson, A.; Loose, M.; Patient, R. The roles of GATA-4, -5 and -6 in vertebrate heart development. Semin. Cell Dev. Biol. 2005, 16, 83-94. [CrossRef] [PubMed]

31. Pourrajab, F.; Babaei Zarch, M.; Baghi Yazdi, M.; Rahimi Zarchi, A.; Vakili Zarch, A. Application of stem cell/growth factor system, as a multimodal therapy approach in regenerative medicine to improve cell therapy yields. Int. J. Cardiol. 2014, 173, 12-19. [CrossRef] [PubMed]

32. Jackson, R.; Tilokee, E.L.; Latham, N.; Mount, S.; Rafatian, G.; Strydhorst, J.; Ye, B.; Boodhwani, M.; Chan, V.; Ruel, M.; et al. Paracrine engineering of human cardiac stem cells with insulin-like growth factor 1 enhances myocardial repair. J. Am. Heart Assoc. 2015, 4, e002104. [CrossRef] [PubMed]

33. Ye, L.; Zhang, S.; Greder, L.; Dutton, J.; Keirstead, S.A.; Lepley, M.; Zhang, L.; Kaufman, D.; Zhang, J. Effective cardiac myocyte differentiation of human induced pluripotent stem cells requires vegf. PLoS ONE 2013, 8, e53764. [CrossRef] [PubMed]

34. Tang, Y.L.; Zhu, W.; Cheng, M.; Chen, L.; Zhang, J.; Sun, T.; Kishore, R.; Phillips, M.I.; Losordo, D.W.; Qin, G. Hypoxic preconditioning enhances the benefit of cardiac progenitor cell therapy for treatment of myocardial infarction by inducing CXCR4 expression. Circ. Res. 2009, 104, 1209-1216. [CrossRef] [PubMed]

35. Tang, Y.L.; Shen, L.; Qian, K.; Phillips, M.I. A novel two-step procedure to expand cardiac Sca-1 + cells clonally. Biochem. Biophys. Res. Commun. 2007, 359, 877-883. [CrossRef] [PubMed]

(C) 2016 by the authors; licensee MDPI, Basel, Switzerland. This article is an open access article distributed under the terms and conditions of the Creative Commons Attribution (CC-BY) license (http:/ / creativecommons.org/licenses/by/4.0/). 